\title{
Impact of Uncertainty Quantification on Design Decisions for a Hydraulic-Hybrid Powertrain Engine
}

\author{
Michael Kokkolaras* \\ Department of Mechanical Engineering, University of Michigan, Ann Arbor, Michigan, 48109 \\ Zissimos P. Mourelatos ${ }^{\dagger}$ \\ Department of Mechanical Engineering, Oakland University, Rochester, Michigan, 48309 \\ and \\ Panos Y. Papalambros ${ }^{\ddagger}$ \\ Department of Mechanical Engineering, University of Michigan, Ann Arbor, Michigan, 48109
}

\begin{abstract}
The method for solving an optimal design problem under uncertainty depends on how the latter is quantified. When sufficient information is available the popular probabilistic approach can (and should) be adopted. In reality however, we often do not have sufficient data to infer appropriate probability distributions for the uncertain quantities modeled as random variables. The amount of available information about the uncertain quantities may be limited to ranges of values (intervals). In this case, the interval analysis approach can be employed to reformulate and solve the optimal design problem. In this study, we use both approaches to solve an engine design optimization problem that considers fuel economy and acceleration performance of a medium-sized truck with a hydraulic-hybrid powertrain. We then contrast the obtained results and comment on the characteristics and features of the two approaches. We also demonstrate an extension of the interval analysis approach to multilevel systems using a simple yet illustrative engine-related example.
\end{abstract}

\section{Introduction}

$\mathrm{T}$ HE general system optimal design model consists of performance objective(s) that must be maximized subject to constraints. Applying mathematical optimization algorithms to solve the associated, generally nonlinear, programming problem typically results in design solutions that lie on the boundaries of constraints. That means that performance cannot be further enhanced unless the constraint boundary is (re)moved. This fact has an additional implication when uncertainty is present and/or the optimal design cannot be realized exactly: deviations from the optimal solution are more likely than not to cause infeasibility, i.e., violate the constraints. Therefore, it is necessary to account for these variations due to uncertainty and adjust the optimal design such that the constraints are not violated when variations occur. In other words, when the designs are pushed to their performance limits, we must make sure that the presence of uncertainty has no adverse impacts.

Uncertainty is inherently present in simulation-based design of complex engineering systems. The analysis models used for the simulation depend on assumptions and include many approximations and empirical constants. Also, advanced yet relatively immature technologies are often associated with uncertainty; the designer is not sure about the validity of the decisions he/she has made, and would like to be able to perform optimization studies under uncertainty. It is therefore imperative to represent uncertainties and take them into account during the early design assessment process.

Uncertainty identification, representation, and quantification are the cornerstones of design optimization under uncertainty. Given the design model and the necessary analysis/simulation models, the designer must first identify all possible sources of uncertainty. Then, she/he must choose an appropriate means to represent and quantify them.

\footnotetext{
* Associate Research Scientist, Department of Mechanical Engineering, University of Michigan, AIAA Member

${ }^{\dagger}$ Associate Professor, Department of Mechanical Engineering, Oakland University, AIAA Member

${ }^{\ddagger}$ Professor, Department of Mechanical Engineering, University of Michigan, AIAA Senior Member
} 
A highly popular approach is to model uncertain quantities as random variables, and quantify them using probability distributions. This approach is quite useful when there are sufficient data to infer appropriate (correct) probability distributions. However, in many situations the designer does not have the necessary information available. In this case, he/she must assume that an uncertain quantity can take any value within a range, and use an interval analysis approach to solve the design optimization problem.

In this study we use both approaches to study the impact of uncertainty representation and quantification on design decisions. We also propose a formulation for extending the interval analysis approach to optimal design of multilevel systems.

The paper is organized as follows. The considered design optimization problem is described in the next section. The two approaches and the corresponding results are presented in Section III. We present the multilevel interval analysis approach in Section IV. Concluding remarks are presented in Section V.

\section{Optimal Design Problem Formulation}

We consider the optimal design problem of a medium-sized dual-use truck with a hydraulic-hybrid powertrain configuration and a fuel cell auxiliary power unit. The design optimization problem is formulated as

$$
\begin{aligned}
& \max _{\mathbf{x}} f(\mathbf{x}, \mathbf{p}) \\
& \text { subject to } \mathbf{g}(\mathbf{x}, \mathbf{p}) \leq \mathbf{0},
\end{aligned}
$$

where the objective function $f$ and the design constraints $\mathbf{g}$ depend on variables $\mathbf{x}$ and parameters $\mathbf{p}$. The objective is to maximize fuel economy (measured in miles per gallon) subject to performance constraints on the acceleration and maximum speed (on different grades) capabilities of the truck. Preliminary optimization studies have indicated that the acceleration constraint is dominates the maximum speed constraints. Therefore, in the remainder of this document, we will focus on the former. Specifically, we require that the time necessary to accelerate from 0 to 45 miles per hour does not exceed 24 seconds.

The objective and the constraint are evaluated using simulation tools. The 6x6 all-wheel-drive truck (powered by a $246 \mathrm{~kW}$ six-cylinder, turbocharged, intercooled, direct injection diesel engine) is modeled using VESIM, a highfidelity vehicle/engine simulation environment and has a gross vehicle weight of $15,300 \mathrm{~kg}^{1}$. A detailed description of the truck model (including the embedded hydraulic system) is given in Assanis et al. ${ }^{2}$ The driving cycle used for the simulation is depicted in Figure 1. The total simulation real-time amounts to 2200 seconds, and corresponds to a distance of approximately $25 \mathrm{~km}$ on a network of primary, secondary, and cross-country roads.

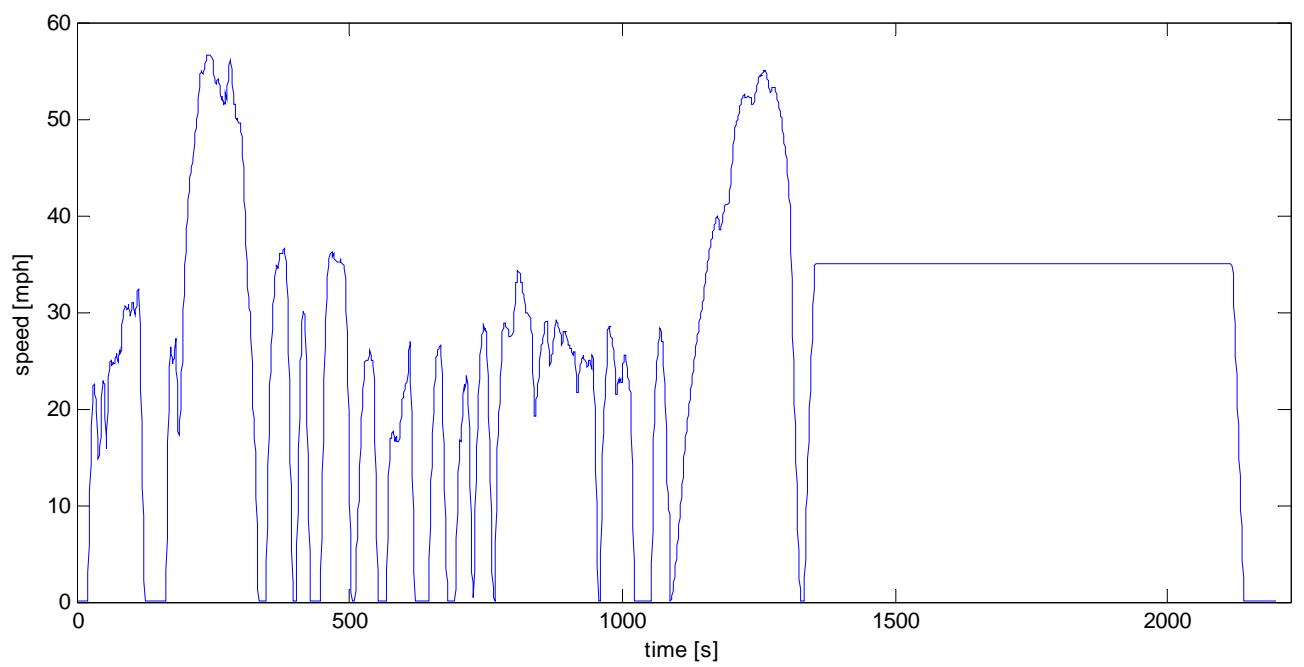

Figure 1. Driving cycle

The inputs list of the integrated VESIM simulation model includes a large number of quantities related to the hybrid powertrain and vehicle dynamics. The designer has to decide which of those inputs to consider as design optimization variables $\mathbf{x}$. The rest are held fixed to pre-specified values, and are considered as parameters $\mathbf{p}$. In this paper we focus on the engine design. Although the hydraulic system design affects the performance of the hybrid 
powertrain, it is not included in this optimization study because the acceleration constraint must be satisfied assuming that only the engine is driving the vehicle. Therefore, we use the optimal hydraulic system design computed in a previous deterministic optimization study ${ }^{3}$. In Kokkolaras et al. ${ }^{4}$ four engine design variables were selected to represent the engine design, and four parameters with the highest impact on the truck's fuel economy and performance were identified among tens of quantities that constitute inputs to the engine and vehicle dynamics simulation codes using the technique of Mohanty and $\mathrm{Wu}^{5}$. These design variables and parameters are summarized in Table 1. They are all considered uncertain in the design optimization and assessment case study presented in this paper.

Table 1. Uncertain design variables and parameters

\begin{tabular}{|l|c|c|c|}
\hline \multicolumn{1}{|c|}{ Uncertain quantity } & Type & Lower bound & Upper bound \\
\hline Displacement [L] & variable & 6 & 9 \\
\hline Compression ratio [-] & variable & 12 & 17 \\
\hline Boost pressure [bar] & variable & 1.87 & 2.55 \\
\hline Wastegate activation speed [RPM] & variable & 1000 & 1600 \\
\hline Injection timing [ ${ }^{\circ}$ ATDC] & parameter & 348 & 350 \\
\hline Frontal area [m ${ }^{2}$ ] & parameter & 6 & 9 \\
\hline Transmission efficiencies [-] & parameter & 0.90 & 0.98 \\
\hline Differential and rolling resistance scaling parameters [-] & parameter & 0.8 & 1.2 \\
\hline
\end{tabular}

\section{Uncertainty Quantification and Corresponding Methods}

In the presence of uncertainty, the formulation of the design optimization problem (1) must be modified. In this section we consider two different approaches for formulating and solving the optimal design problem depending on how uncertainty is represented and quantified. Note that uncertain quantities are denoted by upper case symbols in both approaches.

\section{A. Probabilistic Approach}

In this approach, we assume that we have sufficient data to infer correct probability distributions for the random variables that model the uncertain quantities. We use the means of the random design variables as optimization variables, and maximize the expected value of the objective function, approximated by the objective function evaluated at the means of the random design variables and parameters. Note that we use the terms random design variables and random design parameters to refer to design variables and design parameters, respectively, that are uncertain and modeled as random variables. The design constraints are reformulated as probabilistic, and their satisfaction is requested at a pre-specified reliability level $R$. Assuming, without lack of generality, that all design variables and parameters are random, the design optimization problem (1) is reformulated as

$$
\begin{aligned}
& \max _{\boldsymbol{\mu}_{\mathbf{X}}} \quad E[f(\mathbf{X}, \mathbf{P})] \approx f\left(\boldsymbol{\mu}_{\mathbf{X}}, \boldsymbol{\mu}_{\mathbf{P}}\right) \\
& \text { subject to } \operatorname{Pr}\left[g_{j}(\mathbf{X}, \mathbf{P}) \leq 0\right] \geq R_{j}, \quad j=1, \ldots, J .
\end{aligned}
$$

Note that in our case study $J=1$.

Problem (2) can be solved using any of the available methods for reliability-based design optimization (RBDO), whose name is inspired by the necessity to assess the reliability of satisfying the probabilistic design constraint. We have used a so-called single-loop method ${ }^{6}$ to solve the problem for several reliability levels ${ }^{4}$.

All random design variables and parameters were assumed to be normally distributed (the effect of probability distribution type on design decisions was investigated in Gunawan et al. ${ }^{7}$ ). The standard deviations of the random design variables and the means and standard deviations of the random parameters are summarized in Table 2. 
Table 2. Standard deviations for design variables and means and standard deviations for parameters

\begin{tabular}{|l|c|l|c|c|}
\hline \multicolumn{1}{|c|}{ Variable } & $\sigma$ & \multicolumn{1}{|c|}{ Parameter } & $\mu$ & $\sigma$ \\
\hline Displacement [L] & 0.025 & Injection timing [ ${ }^{\circ}$ ATDC] & 350 & 1 \\
\hline Compression ratio [-] & 0.1 & Frontal area [m ${ }^{2}$ ] & 7.5 & 0.75 \\
\hline Boost pressure [bar] & 0.1 & Transmission efficiencies [-] & 0.94 & 0.02 \\
\hline Wastegate activation speed [RPM] & 50 & $\begin{array}{l}\text { Differential and rolling resistance scaling } \\
\text { parameters [-] }\end{array}$ & 1.0 & 0.1 \\
\hline
\end{tabular}

Table 3 summarizes the results for different levels of required reliability. These results are presented in more detail in Kokkolaras et al. ${ }^{4}$; they are included here to enable the contrast to the results obtained using the interval analysis approach. It can be seen that the engine size increases, while compression ratio, and boost pressure decrease with increasing level of desired reliability of acceleration performance, which is, of course, detrimental to fuel economy. An improvement of 6.6\% performance reliability (from 93.3\% to 99.9\%) induces a $7.6 \%$ fuel economy reduction (from $6.69 \mathrm{mpg}$ to $6.18 \mathrm{mpg}$ ). It can also be seen that we must sacrifice slightly less optimality to improve performance reliability from $93.3 \%$ to $99.3 \%$ than from $99.3 \%$ to $99.9 \%$, i.e., the need for performance sacrifice increases nonlinearly as we demand higher reliability.

Table 3. Optimal designs for different levels of reliability

\begin{tabular}{|l|c|c|c|c|c|c|c|}
\hline $\begin{array}{l}\text { Expected objective function value (fuel } \\
\text { economy in mpg) }\end{array}$ & 6.69 & 6.62 & 6.54 & 6.44 & 6.35 & 6.29 & 6.18 \\
\hline $\begin{array}{l}\text { Reliability level of satisfying the } \\
\text { acceleration constraint in \% }\end{array}$ & 93.3 & 96.4 & 98.2 & 99.3 & 99.7 & 99.8 & 99.9 \\
\hline Mean engine displacement in L & 6.5 & 6.8 & 7.1 & 7.6 & 7.9 & 8.2 & 8.7 \\
\hline Mean engine compression ratio & 16.85 & 16.82 & 16.79 & 16.75 & 16.72 & 16.70 & 16.67 \\
\hline Mean boost pressure in bar & 2.40 & 2.37 & 2.34 & 2.30 & 2.27 & 2.25 & 2.22 \\
\hline Mean wastegate activation speed in RPM & 1200 & 1200 & 1200 & 1200 & 1200 & 1200 & 1200 \\
\hline
\end{tabular}

\section{B. Interval Analysis Approach}

The probabilistic approach is very useful and should be adopted when the designer has sufficient data to model uncertain quantities as random variables with appropriate probability distributions. When this is not the case, it is imperative to assume that the uncertain quantities can take any value within a range. Note that this not equivalent to assuming a uniform distribution as it does not imply that the probability of taking a specific value in a range is equal to any other value within that range.

We view the interval analysis approach as a possibility theory case ${ }^{8,9}$ where information availability is limited to a minimum. Designs obtained using possibility-based design optimization (PBDO) methods are typically conservative compared to the ones obtained using RBDO methods. Possibility-based designs sacrifice additional optimality compared to RBDO designs to account for lack of uncertainty information and avoid constraint violation.

According to possibility theory, the possibility $\pi(A)$ of event $A$ provides an upper bound on the probability $P(A)$, i.e., $P(A) \leq \pi(A)$. From the design point of view, we can conclude that what is possible may not be probable, and what is impossible is also improbable. If the possibility of violating a constraint is zero, then the probability of violating the same constraint will also be zero. If feasibility of a constraint $g$ is expressed with the negative null form $g \leq 0$ as in Problem (1), the constraint is always satisfied if $\pi(g>0)=0$. By introducing the notion of membership functions and $\alpha$-cuts, we can relax this requirement as $\pi(g>0) \leq \alpha$, provided that $0<\alpha<<1^{10}$. According to possibility theory, Problem (1) is reformulated as 


$$
\begin{aligned}
& \max _{\mathbf{X}_{\mathrm{N}}} f(\mathbf{X}, \mathbf{P}) \\
& \text { subject to } \pi\left[g_{j}(\mathbf{X}, \mathbf{P})>0\right] \leq \alpha, \quad 0<\alpha \leq 1, \quad j=1, \ldots, J .
\end{aligned}
$$

It can then be shown ${ }^{11}$ that if the maximum possibly attainable value of the constraint $g$ at the corresponding $\alpha$ cut is less than or equal to zero, i.e., $g_{\max }^{\alpha} \leq 0$, the possibility of violating this constraint is less than $\alpha$. In general, membership functions express how ranges of values that bound the uncertainty quantities are decreased with increasing amount of information. The $\alpha$-cuts denote levels of information, starting at the lowest $(\alpha=0)$, where the range is largest, and increasing to the highest $(\alpha=1)$, where the range is the smallest (possibly a crisp value). In this work, we will assume that the lowest level of information is available, where $\alpha$ is equal to zero. Therefore, we do not have to consider membership functions and higher $\alpha$-cuts. Thus, the possibility-based design optimization problem (3) boils down to an interval analysis-based optimization problem formulated as

$$
\begin{aligned}
& \max _{\mathbf{X}_{\mathbf{N}}} f(\mathbf{X}, \mathbf{P}) \\
& \text { subject to } g_{j, \max } \leq 0, \quad j=1, \ldots, J .
\end{aligned}
$$

The implementation process of solving Problem (4) involves a nested optimization. The outer-loop optimization determines optimal nominal values $\mathbf{X}_{\mathbf{N}}$ for the uncertain design variables $\mathbf{X}$. For each iterate of $\mathbf{X}_{\mathbf{N}}$, inner-loop optimization problems are solved, one for the objective and one for each constraint. Specifically, worst-case scenario problems are formulated, in which the uncertain design variables and parameters are treated as optimization variables. These worst-case optimization problems (also referred to as "anti-optimization" problems ${ }^{12}$ ) may involve a larger number of optimization variables, but are only bound-constrained. The bounds of the inner-loop optimization problem variables are fixed for the uncertain parameters, while they are determined utilizing uncertainty information around the current nominal values for the design variables. The inner-loop optimization problem for the objective is formulated as

$$
\begin{array}{ll}
\min _{\mathbf{X}, \mathbf{P}} & f(\mathbf{X}, \mathbf{P}) \\
\text { subject to } & \mathbf{X}_{\mathbf{N}}-\boldsymbol{\Delta} \mathbf{X} \leq \mathbf{X} \leq \mathbf{X}_{\mathbf{N}}+\Delta \mathbf{X} \\
& \mathbf{P}_{\min } \leq \mathbf{P} \leq \mathbf{P}_{\max }
\end{array}
$$

and for each constraint $j$ as

$$
\begin{array}{cc}
\max _{\mathbf{X}, \mathbf{P}} & g_{j}(\mathbf{X}, \mathbf{P}) \\
\text { subject to } & \mathbf{X}_{\mathbf{N}}-\mathbf{\Delta X} \leq \mathbf{X} \leq \mathbf{X}_{\mathbf{N}}+\mathbf{\Delta X} \\
& \mathbf{P}_{\min } \leq \mathbf{P} \leq \mathbf{P}_{\max } .
\end{array}
$$

The primary purpose of solving problems (5) and (6) is not to determine optimal values for $\mathbf{X}$ and $\mathbf{P}$, but to obtain the minimal value of $f$ and maximal value of $g$ that may be attained due to uncertainty in $\mathbf{X}$ and $\mathbf{P}$ (note that we assume "homogeneous" uncertainty, i.e., that $\Delta \mathbf{X}$ does not vary with $\mathbf{X}_{\mathrm{N}}$ ). These two values are used in the outerloop optimization, where the worst objective value is maximized and the worst constraint value must be feasible. Nevertheless, the inner-loop optimal values of $\mathbf{X}$ and $\mathbf{P}$ can be used to draw conclusions about how to attempt to control uncertainty, i.e., what values to desire and what values to avoid, if possible.

The nested optimization problems (4)-(6) have been solved for our design case study using the uncertainty information summarized in Table 4, where $\gamma$ is used as a parameter to represent larger or smaller ranges of uncertainty. To facilitate the contrast of results, the uncertainty ranges of the design variables in Table 4 for $\gamma=1$ and $\gamma=2$ correspond to one- and two- $\sigma$ deviations from the nominal point, respectively, where $\sigma$ is the standard deviation in the case of normally distributed random design variables (Table 2). Similarly, the design parameter ranges (Table 4) are chosen to be comparable in size with the normally distributed design parameters of Table 2 (approximately 95\% of the values of the normally distributed parameters will lie within the ranges of Table 4). 
Table 4. Ranges of design variables and parameters

\begin{tabular}{|l|c|l|c|}
\hline \multicolumn{1}{|c|}{ Variable } & \multicolumn{1}{c|}{ Range } & \multicolumn{1}{c|}{ Parameter } & Range \\
\hline Displacement [L] & nominal $+/-\gamma * 0.025$ & Injection timing [ ${ }^{\circ}$ ATDC] & $348-352$ \\
\hline Compression ratio [-] & nominal $+/-\gamma^{*} 0.1$ & Frontal area [m ${ }^{2}$ ] & $6.0-9.0$ \\
\hline Boost pressure [bar] & nominal $+/-\gamma^{*} 0.1$ & Transmission efficiencies [-] & $0.90-0.98$ \\
\hline $\begin{array}{l}\text { Wastegate activation speed } \\
\text { [RPM] }\end{array}$ & nominal $+/-\gamma^{*} 50$ & $\begin{array}{l}\text { Differential and rolling resistance } \\
\text { scaling parameters [-] }\end{array}$ & $0.8-1.2$ \\
\hline
\end{tabular}

The derivative-free, global search optimization algorithm DIRECT ${ }^{13}$ ) was used to maximize the likelihood of converging to global optima for both the outer and the inner optimization problems. The optimal designs corresponding to two different uncertainty ranges $(\gamma=1$ and $\gamma=2)$ are reported in Table 5.

Table 5. Optimal designs for different values of $\gamma$

\begin{tabular}{|l|c|c|}
\hline \multicolumn{1}{|c|}{ Variable } & Nominal value, $\gamma=1$ & Nominal value, $\gamma=2$ \\
\hline Displacement [L] & 6.55 & 7.57 \\
\hline Compression ratio [-] & 16.87 & 16.77 \\
\hline Boost pressure [bar] & 2.45 & 2.34 \\
\hline Wastegate activation speed [RPM] & 1158 & 1226 \\
\hline
\end{tabular}

The optimal designs have been obtained considering the worst-case scenarios for both the objective and the constraint. Therefore, Problems (5) and (6) must be solved one more final time with reversed objectives (max and min, respectively) to obtain the best-case scenario values for the objective and the constraint, and thus the complete ranges of possibly attainable values of these two functions given the uncertainty in $\mathbf{X}$ and $\mathbf{P}$.

Tables 6 and 7 include the ranges obtained for the objective and the constraint and the associated design variable and parameter values for $\gamma=1$ and $\gamma=2$, respectively. This information can be quite useful to the designer. For example, it can be seen that the same set of parameter values are adversary or beneficial to both fuel economy and performance. Thus, if the designer can allocate resources to reduce or control uncertainty, he/she knows which parameter values to strive for. On the other side, it can be observed that engine displacement and wastegate activation speed extremes benefit or degrade either fuel economy or performance, but not both simultaneously, while engine compression ratio and boost pressure extremes benefit or degrade fuel economy and performance simultaneously.

It can also be seen that the uncertainty range has a significant impact on both fuel economy and acceleration performance. As the uncertainty ranges of the simulation inputs (design variables and parameters) increase (from $\gamma=$ 1 to $\gamma=2$ ), the uncertainty ranges of the simulation outputs (objective and constraint function values) also increase. Fuel economy varies between 6.06 and $7.34 \mathrm{mpg}$ for $\gamma=1$ and between 5.78 and $7.13 \mathrm{mpg}$ for $\gamma=2$. The worst-case values are $17.4 \%$ and $18.9 \%$ worse than the best-case values, respectively. The $0-45 \mathrm{mph}$ acceleration time varies between 18 and 24 seconds for $\gamma=1$ and between 16 and 24 seconds for $\gamma=2$. The worst-case values are $25 \%$ and $33.3 \%$ worse than the best-case values, respectively. Note that the enlargement of the uncertainty ranges of the simulation outputs is not symmetric. The minimum possibly attainable fuel economy value decreases (from 6.06 mpg to $5.78 \mathrm{mpg}$ ), while the maximum possibly attainable value does not increase (it also decreases from $7.34 \mathrm{mpg}$ to $7.13 \mathrm{mpg}$ ). The worst-case scenario constraint value is 24 seconds in both cases (i.e., the constraint is active), but the "higher-uncertainty" optimal design yields an improved best-case acceleration value due to the engine up-sizing. 
Table 6. Objective and constraint ranges and associated uncertain design variable and parameter values for $\gamma=1$

\begin{tabular}{|l|c|c|c|c|c|c|c|c|c|}
\hline & Value & $\begin{array}{c}\text { Displ. } \\
{[\mathrm{L}]}\end{array}$ & $\begin{array}{c}\text { Compr. } \\
\text { ratio }[-]\end{array}$ & $\begin{array}{c}\text { Boost } \\
\text { press. } \\
\text { [bar] }\end{array}$ & $\begin{array}{c}\text { Wast. } \\
\text { act. sp. } \\
\text { [RPM] }\end{array}$ & $\begin{array}{c}\text { Inj. tim. } \\
{\left[{ }^{\circ} \mathrm{ADTC}\right]}\end{array}$ & $\begin{array}{c}\text { Area } \\
{\left[\mathrm{m}^{2}\right]}\end{array}$ & $\begin{array}{c}\text { Trans. } \\
\text { eff. [-] }\end{array}$ & $\begin{array}{c}\text { Rolling } \\
\text { res./diff. } \\
\text { scal. [-] }\end{array}$ \\
\hline$f_{\min }$ & $\begin{array}{c}6.06 \\
\mathrm{mpg}\end{array}$ & 6.575 & 16.77 & 2.35 & 1108 & 352 & 9 & 0.90 & 1.2 \\
\hline$f_{\max }$ & $\begin{array}{c}7.34 \\
\mathrm{mpg}\end{array}$ & 6.525 & 16.97 & 2.55 & 1208 & 348 & 6 & 0.98 & 0.8 \\
\hline$g_{\min }$ & $18 \mathrm{~s}$ & 6.575 & 16.97 & 2.55 & 1108 & 348 & 6 & 0.98 & 0.8 \\
\hline$g_{\max }$ & $24 \mathrm{~s}$ & 6.525 & 16.77 & 2.35 & 1208 & 352 & 9 & 0.90 & 1.2 \\
\hline
\end{tabular}

Table 7. Objective and constraint ranges and associated uncertain design variable and parameter values for $\gamma=\mathbf{2}$

\begin{tabular}{|l|c|c|c|c|c|c|c|c|c|}
\hline & Value & $\begin{array}{c}\text { Displ. } \\
{[\mathrm{L}]}\end{array}$ & $\begin{array}{c}\text { Compr. } \\
\text { ratio }[-]\end{array}$ & $\begin{array}{c}\text { Boost } \\
\text { press. } \\
\text { [bar] }\end{array}$ & $\begin{array}{c}\text { Wast. } \\
\text { act. sp. } \\
{[\text { RPM] }}\end{array}$ & $\begin{array}{c}\text { Inj. tim. } \\
{\left[{ }^{\circ} \text { ADTC] }\right.}\end{array}$ & $\begin{array}{c}\text { Area } \\
{\left[\mathrm{m}^{2}\right]}\end{array}$ & $\begin{array}{c}\text { Trans. } \\
\text { eff. [-] }\end{array}$ & $\begin{array}{c}\text { Rolling } \\
\text { res./diff. } \\
\text { scal. [-] }\end{array}$ \\
\hline$f_{\min }$ & $\begin{array}{c}5.78 \\
\mathrm{mpg}\end{array}$ & 7.62 & 16.57 & 2.14 & 1126 & 352 & 9 & 0.90 & 1.2 \\
\hline$f_{\max }$ & $\begin{array}{c}7.13 \\
\mathrm{mpg}\end{array}$ & 7.52 & 16.97 & 2.54 & 1326 & 348 & 6 & 0.98 & 0.8 \\
\hline$g_{\min }$ & $16 \mathrm{~s}$ & 7.62 & 16.97 & 2.54 & 1126 & 348 & 6 & 0.98 & 0.8 \\
\hline$g_{\max }$ & $24 \mathrm{~s}$ & 7.52 & 16.57 & 2.14 & 1326 & 352 & 9 & 0.90 & 1.2 \\
\hline
\end{tabular}

\section{Remarks}

By inspecting Tables 3 and 5, we observe that increasing the reliability level in the probabilistic approach and the uncertainty range in the interval analysis approach have a similar effect on design decisions. They cause engine displacement and wastegate activation speed to increase, and compression ratio and boost pressure to decrease. The optimal design of the $\gamma=1$ case in the interval analysis approach is very close to the optimal design of the 93.3\% reliability level in the probabilistic approach, while the optimal design of the $\gamma=2$ case in the interval analysis approach is very close to the optimal design of the $99.3 \%$ reliability level in the probabilistic approach. The approximate expected fuel economy values in the probabilistic approach for the optimal designs at the 93.3\% and 99.3\% reliability levels are the midpoints of the fuel economy ranges of the corresponding optimal designs in the interval analysis approach.

The probabilistic approach assures that design constraints will not be violated according to a pre-specified reliability level, while the interval analysis approach excludes the possibility of constraint violation at the expense of a more conservative design. The interval analysis approach provides the range of possibly attainable constraint values. Using the probabilistic approach, we can obtain statistical properties on the constraint functions, but at an additional computational cost.

Both approaches face similar challenges regarding accuracy and efficiency. In the probabilistic approach, there is a trade-off between accuracy and computational efficiency when performing the reliability analysis of the probabilistic constraints. Analytical methods are more efficient but less accurate than simulation-based (Monte Carlo) methods. Note that when problems are highly nonlinear, analytical methods that compute most probable points (MPP's) may encounter difficulties.

The interval analysis approach requires finding the global optimum of the inner-loop problems. The likelihood of finding a global optimum is increased only by more elaborate exploration of the design space. Thus, a similar accuracy versus efficiency trade-off exists. It is encouraging that both the reliability analysis of the probabilistic constraints and the solution of the inner-loop optimization problems can be performed in parallel. A possible advantage of the interval analysis approach is that the inner-loop optimization problems are only bound-constrained, i.e., there are no general nonlinear constraints, which makes their solution much more tractable. 
Finally, the fact that, besides the design variables, many design parameters may be uncertain increases the size of the inner-loop problems for both approaches.

\section{Extension of the Interval Analysis Approach to Multilevel Systems}

Optimal system design problems are often formulated in multiple levels, either because they are decomposed hierarchically, or due to the structure of the required simulations. In this section, we present an extension of the interval analysis approach to optimal design of multilevel systems under uncertainty. Our objective is to demonstrate the presented formulation using a simple yet illustrative example; a detailed discussion of deterministic and nondeterministic design of hierarchical multilevel systems is out of the scope of this paper. We will use the bi-level, engine-related, simulation-based example from Kokkolaras et al. ${ }^{14}$, where we proposed a probabilistic approach for optimal design of hierarchically decomposed, multilevel systems under uncertainty.

We consider a V6 gasoline engine as the top-level system, which is decomposed into a bottom-level subsystem that represents the piston-ring/cylinder-liner subassembly of six identical cylinders (thus, we only need to consider one). The bottom-level (ring/liner subassembly) simulation takes as inputs the surface roughness of the ring and the liner and the Young's modulus and hardness of the liner, and computes power loss due to friction, oil consumption, blow-by, and liner wear rate. The top-level (engine) simulation takes as input power loss due to friction and computes fuel consumption (cf. Figure 2).

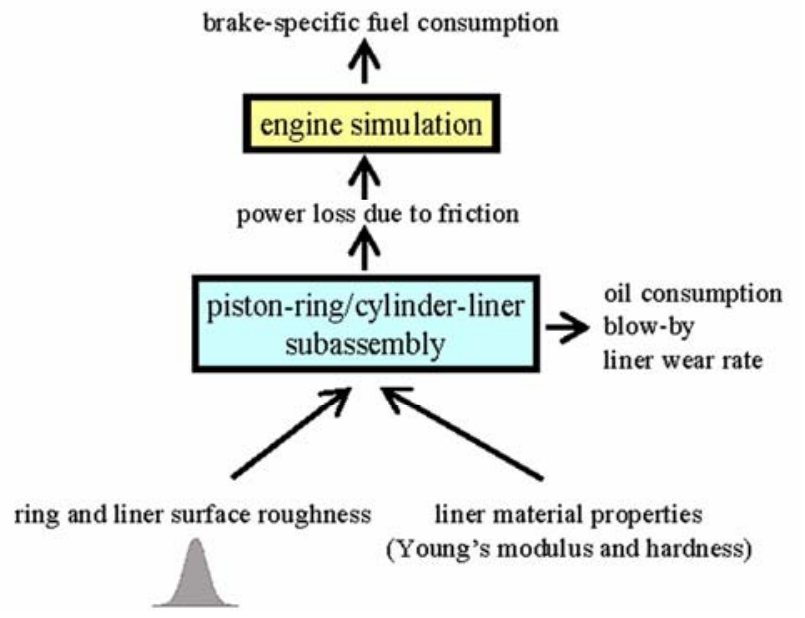

Figure 2. Bi-level system simulation of engine and ring/liner

The considered uncertain quantities are ring and liner surface roughnesses; root mean square (RMS) of asperity height is used to represent and quantify surface roughness. Of course, liner material properties can (and should) also be considered as uncertain, but we have not done so in this example to keep it simple. Let us assume that surface roughness $X$ exhibits deviations from nominal values that can be quantified by an interval of the form $\left[X_{\mathrm{N}^{\mathrm{N}}}-\delta_{X} X_{\mathrm{N}}\right.$, $X_{\mathrm{N}}+\delta_{X} X_{\mathrm{N}}$ ], where $\delta_{X}$ denotes percentage deviation. This surface roughness interval uncertainty is propagated through the simulation hierarchy to estimate intervals for power loss and fuel consumption. Since uncertainty information is available at the bottom-level we first formulate and solve the bottom-level problem

$$
\begin{array}{ll}
\min _{X_{1, \mathrm{~N}}, X_{2, \mathrm{~N}}, x_{3}, x_{4}} & \left(P L_{\mathrm{B}}\left(X_{1}, X_{2}, x_{3}, X_{4}\right)-P L_{\mathrm{B}}^{\mathrm{U}}\right)^{2}+\left(P L_{\mathrm{W}}\left(X_{1}, X_{2}, X_{3}, x_{4}\right)-P L_{\mathrm{W}}^{\mathrm{U}}\right)^{2} \\
\text { subject to } & O C_{\max }\left(X_{1}, X_{2}, x_{3}, X_{4}\right) \leq 15.3 \times 10^{-3} \mathrm{~kg} / \mathrm{hr} \\
& B B_{\max }\left(X_{1}, X_{2}, X_{3}, x_{4}\right) \leq 4.25 \times 10^{-5} \mathrm{~kg} / \mathrm{s} \\
& W R_{\max }\left(X_{1}, X_{2}, X_{3}, x_{4}\right) \leq 2.4 \times 10^{-12} \mathrm{~m}^{3} / \mathrm{s} \\
& 2 \mu \mathrm{m} \leq X_{1, \mathrm{~N}} \leq 9 \mu \mathrm{m}, 2 \mu \mathrm{m} \leq X_{2, \mathrm{~N}} \leq 9 \mu \mathrm{m}, \\
& 80 \mathrm{GPa} \leq x_{3} \leq 340 \mathrm{GPa}, 150 \mathrm{BHV} \leq x_{4} \leq 240 \mathrm{BHV},
\end{array}
$$

where $X_{1}$ and $X_{2}$ are (uncertain) ring and liner surface roughness design variables, respectively, $x_{3}$ and $x_{4}$ are (deterministic) liner Young's modulus and hardness design variables, respectively, $P L$ is power loss due to friction 
(subscripts "W" and "B" denote worst and best possible values due to interval uncertainty, respectively, while superscript " $U$ " denotes target value from the upper level), $O C$ denotes oil consumption, $B B$ denotes blow-by, and $W R$ denotes liner wear rate. According to the interval analysis approach, at the outer-loop optimization we determine nominal values $X_{1, \mathrm{~N}}$ and $X_{2, \mathrm{~N}}$ (as well as optimal values for $x_{3}$ and $x_{4}$ ), while solving five (5) inner-loop optimization problems given the (assumed invariant) surface roughness interval uncertainty: one best-case scenario for the power loss, one worst-case scenario for the power loss, and one worst-case scenario each for oil consumption, blow-by, and wear rate. Since we do not have information from the top-level problem yet, i.e., target values for $P L_{\mathrm{B}}$ and $P L_{\mathrm{W}}$, we assume these to be equal to zero. Once the power loss uncertainty interval has been obtained, we compute percentage deviation $\delta_{P L}$ from the interval midpoint in order to pass this uncertainty information to the top-level problem, which is formulated as

$$
\min _{P L_{\mathrm{N}}}\left(F C_{\mathrm{B}}(P L)-F C_{\mathrm{B}}^{\mathrm{T}}\right)^{2}+\left(F C_{\mathrm{W}}(P L)-F C_{\mathrm{W}}^{\mathrm{T}}\right)^{2}+w\left(P L_{\mathrm{B}}-P L_{\mathrm{B}}^{\mathrm{L}}\right)^{2}+w\left(P L_{\mathrm{W}}-P L_{\mathrm{W}}^{\mathrm{L}}\right)^{2},
$$

where FC denotes fuel consumption. The superscript "T" denotes target values, while the superscript "L" denotes interval values from the lower level, so that the top-level problem does not consider solutions that are too far from what the bottom-level can provide (the weight $w$ can be adjusted to emphasize consistency rather than fuel consumption optimality). At the outer-loop optimization we determine nominal values of power loss while solving two inner-loop optimization problems given the quantified (at the lower level) power loss interval uncertainty: one best-case scenario for the fuel consumption and one worst-case scenario for the fuel consumption. After the toplevel problem is solved (note that the desired fuel consumption interval target values may not be achieved), the power loss interval is updated, passed down to the bottom-level problem, which is then resolved and so on. We assume that this coordination process is converged when all quantities do not change significantly anymore.

Table 8 reports the results obtained assuming $\delta_{X}=0.1(10 \%)$ for both the ring and the liner surface roughness uncertainty (cells are empty when quantities are not related to a problem; power loss links the two problems). All optimization problems (top and bottom levels, outer and inner loops) were solved using the NOMADm ${ }^{15}$ software package. NOMADm is the Matlab implementation of the Generalized Pattern Search (GPS) and Mesh Adaptive Direct Search (MADS) family of derivative-free algorithms for mixed variable programming ${ }^{16,17}$.

Table 8. Results of the multilevel system design problem using the interval analysis approach

\begin{tabular}{|l|c|c|c|c|c|c|c|c|c|c|}
\hline & $\begin{array}{c}X_{1, \mathrm{~N}} \\
{[\mu \mathrm{m}]}\end{array}$ & $\begin{array}{c}X_{2, \mathrm{~N}} \\
{[\mu \mathrm{m}]}\end{array}$ & $\begin{array}{c}x_{3} \\
{[\mathrm{GPa}]}\end{array}$ & $\begin{array}{c}x_{4} \\
{[\mathrm{BHV}]}\end{array}$ & $\begin{array}{c}P L_{\mathrm{B}} \\
{[\mathrm{kW}]}\end{array}$ & $\begin{array}{c}P L_{\mathrm{W}} \\
{[\mathrm{kW}]}\end{array}$ & $\begin{array}{c}\delta_{\mathrm{PL}} \\
{[\%]}\end{array}$ & $\begin{array}{c}F C_{\mathrm{B}} \\
{[\mathrm{kg} /} \\
\mathrm{kWhr}]\end{array}$ & $\begin{array}{c}F C_{\mathrm{W}} \\
{[\mathrm{kg} /} \\
\mathrm{kWhr}]\end{array}$ & $\begin{array}{c}\delta_{\mathrm{FC}} \\
{[\%]}\end{array}$ \\
\hline Bottom-level solution & 2.06 & 5.87 & 80 & 40 & 0.277 & 0.369 & 15 & - & - & - \\
\hline Top-level solution, $w=1$ & - & - & - & - & 0.176 & 0.238 & 15 & 0.486 & 0.499 & 1.3 \\
\hline Top-level solution, $w=10$ & - & - & - & - & 0.253 & 0.343 & 15 & 0.502 & 0.522 & 2 \\
\hline Top-level solution, $w=1000$ & - & - & - & - & 0.263 & 0.356 & 15 & 0.504 & 0.525 & 2 \\
\hline
\end{tabular}

In order to achieve the best (minimal) fuel consumption possible, we set the top-level problem target values for both the worst and the best fuel consumption equal to zero. Of course, these target values are unattainable. Therefore, the power loss interval computed by solving the bottom-level problem $([0.277,0.369])$ cannot be matched exactly when solving the top-level problem. By increasing the values for the weight $w$, we increase consistency, i.e., interval matching for the power loss $([0.263,0.356]$ for $w=1000)$. It is interesting that while the power loss uncertainty is invariantly quantified at $15 \%$ around the interval midpoint, the fuel consumption uncertainty changes for different weight values (from 1.3\% to $2 \%$ around the interval midpoint). This implies that uncertainty is not invariant with respect to the design point, as assumed in many design under uncertainty methodologies.

\section{Concluding Remarks}

We investigated the impact of uncertainty quantification on design decisions by studying an optimal design problem. Specifically, we contrasted two relatively extreme situations: one where the designer has complete information and data available to infer probability distributions of random variables and adopt a probabilistic design approach, and one where he/she has the lowest amount of information available, and has to assume ranges for the 
uncertain quantities and adopt an interval analysis approach for design optimization. As expected, the interval analysis approach yields design solutions that are conservative relative to the ones obtained using a probabilistic design approach, especially as interval uncertainty increases. However, the interval analysis approach ensures feasibility at all times. In terms of computational cost, the nested optimization of the interval analysis approach seems to be less expensive than the required reliability analysis (analytical or simulation-based) in the probabilistic approach. It is also less challenging numerically since the inner-loop optimization problems are simple boundconstrained problems. The single challenge is that the inner-loop problems require global solutions to ensure consideration of the worst-case scenario. One of the pluses of the interval analysis approach is that the solution of the inner-loop problems provides information to the designer with respect to the beneficial or adversary effects of uncertainty so that, if possible, future resources can be allocated to improve design robustness. Also, if it makes sense, unbounded interval uncertainty, i.e., infinite intervals can be considered in the interval analysis design approach. One of our significant findings is that interval uncertainty does not necessarily propagate either symmetrically or invariantly. Finally, we also presented a first extension of the interval analysis approach to design of multilevel systems. Future work will focus on a more elaborate study of the multilevel interval analysis approach.

\section{Acknowledgments}

The authors would like to acknowledge the support of the Automotive Research Center (ARC), a U.S. Army Center of Excellence for Modeling and Simulation of Ground Vehicles led by the University of Michigan. The first and third authors also acknowledge the support of the NSF through the grant award DMI-0503737. Such support does not constitute an endorsement by the funding agencies of the opinions expressed in the article.

\section{References}

${ }^{1}$ Assanis, D.N., Filipi, Z.S., Gravante, S., Grohnke, D., Gui, X., Louca, L.S., Rideout, G.D., Stein, J.L., and Wang Y., "Validation and Use of SIMULINK Integrated, High Fidelity, Engine-In-Vehicle Simulation of the International Class VI Truck,” SAE Paper 2000-01-0288.

${ }^{2}$ Filipi, Z., Louca, L., Stefanopoulou, A., Pukrushpan, J., Kittirungsi, B., and Peng, H., "Fuel Cell APU for Silent Watch and Mild Electrification of a Medium Tactical Truck," Proceedings of the SAE World Congress, March 2004, Detroit, Michigan, paper no. 2004-01-1477.

${ }^{3}$ Filipi, Z.S., Louca, L.S., Daran, B., Lin, C.-C., Yildir, U., Wu, B., Kokkolaras, M., Assanis, D.N., Peng, H., Papalambros, P.Y., and Stein, J.L., "Combined Optimization of Design and Power Management of the Hydraulic Hybrid Propulsion System for a 6x6 Medium truck,” International Journal of Heavy Vehicle Systems, 11(3-4):371-401, 2004.

${ }^{4}$ Kokkolaras, M., Mourelatos, Z., Louca, L., Filipi, Z., Delagrammatikas, G., Stefanopoulou, A., Papalambros, P., and Assanis, D., "Design under Uncertainty and Assessment of Performance Reliability of a Dual-Use Medium Truck with Hydraulic-Hybrid Powertrain and Fuel Cell Auxiliary Power Unit," Proceedings of the SAE World Congress, April 2005, Detroit, Michigan, paper no. 2005-01-1396.

${ }^{5}$ Mohanty, S. and Wu, Y.T., "CDF Sensitivity Analysis Technique for Ranking Parameters in the Performance Assessment of the Proposed High-Level Waste Repository at Yucca Mountain, Nevada, USA,” Reliability Engineering and System Safety, 73:167-176, 2001.

${ }^{6}$ Liang, J., Mourelatos, Z.P., and Tu, J., “A single-loop method for reliability-based design optimization,” Proceedings of the 30th ASME Design Automation Conference, September 2004, Salt Lake City, Utah, paper no. DAC-57255.

${ }^{7}$ Gunawan, S., Kokkolaras, M., Papalambros, P.Y., and Mourelatos, Z.P., “Optimal Design and Reliability Estimation with Incomplete Uncertainty Information,” Proceedings of the 2006 SAE World Congress, April 3-7, 2006, Detroit, Michigan, paper no. 2006-01-0962.

${ }^{8}$ Dubois, D. and Prade, H., Possibility Theory, Plenum Press, New York, 1988.

${ }^{9}$ Ross, T. J., Fuzzy Logic with Engineering Applications, McGraw Hill, 1995.

${ }^{10}$ Zadeh, L. A., "Fuzzy Sets as a Basis for a Theory of Possibility,” Fuzzy Sets and Systems, 1:3-28, 1978.

${ }^{11}$ Mourelatos Z.P. and Zhou, J., "Reliability Estimation and Design with Insufficient Data Based on Possibility Theory," AIAA Journal, 43(8):1696-1705, 2005.

${ }^{12}$ Elishakoff, I., Haftka, R.T., and Fang, J.J., "Structural Design under Bounded Uncertainty - Optimization with Antioptimization,” International Journal of Computers \& Structures, 53(6):1401-1405, 1994

${ }^{13}$ Jones, D.R., “The DIRECT Global Optimization Algorithm,” Encyclopedia of Optimization, (1):431-440, 2001.

${ }^{14}$ Kokkolaras, M., Mourelatos, Z.P., and Papalambros, P.Y., "Design Optimization of Hierarchically Decomposed Multilevel Systems under Uncertainty,” Transactions of the ASME - Journal of Mechanical Design, 128(2):503-508, 2006.

${ }^{15} \mathrm{M}$. Abramson, NOMADm software and user's guide, http://en.afit.edu/ENC/Faculty/MAbramson/NOMADm.html, last accessed on March 2006.

${ }^{16}$ Audet, C., and Dennis, J.E., Jr., “Analysis of Generalized Pattern Searches,” SIAM Journal on Optimization, 13(3):889-903, 2003.

${ }^{17}$ Audet, C., and Dennis, J.E., Jr., "Mesh Adaptive Direct Search Algorithms for Constrained Optimization,” to appear in SIAM Journal on Optimization. 